2. Touzard-Romo F, Tapé C, Lonks JR. Co-infection with SARS-CoV-2 and human metapneumovirus. R I Med J. 2020;103:75-6. PubMed

3. Wu X, Cai Y, Huang X, Yu X, Zhao L, Wang F, et al. Co-infection with SARS-CoV-2 and influenza A virus in patient with pneumonia, China. Emerg Infect Dis. 2020;26:1324-6. https:/ / doi.org/10.3201/eid2606.200299

4. Richardson S, Hirsch JS, Narasimhan M, Crawford JM, McGinn T, Davidson KW, et al. Presenting characteristics, comorbidities, and outcomes among 5700 patients hospitalized with COVID-19 in the New York City area. JAMA. 2020;323:2052-59. https://doi.org/10.1001/jama.2020.6775

5. Arashiro T, Nakamura S, Asami T, Mikuni H, Fujiwara E, Sakamoto S, et al. SARS-CoV-2 and Legionella co-infection in a person returning from a Nile Cruise. J Travel Med. 2020;27:taaa053. PubMed https://doi.org/10.1093/jtm/taaa053

6. Cox MJ, Loman N, Bogaert D, O'Grady J. Co-infections: potentially lethal and unexplored in COVID-19. Lancet Microbe.2020;1:e11. https://doi.org/10.1016/ S2666-5247(20)30009-4

7. ESCMID. ESCMID Study Group for Legionella Infections [cited 2020 Sep 24]. https:/ / www.escmid.org/research_projects/ study_groups/study_groups_g_n/legionella_infections

8. Public Health England. Legionnaires' disease in residents of England and Wales: 2016 [cited 2020 May 6]. https:/ / www. gov.uk/government/publications/legionnaires-disease-inresidents-of-england-and-wales-2016

9. Adler H, Ball R, Fisher M, Mortimer K, Vardhan MS. Low rate of bacterial co-infection in patients with COVID-19. Lancet Microbe. 2020;1:e62. https:/ / doi.org/10.1016/ S2666-5247(20)30036-7

Address for correspondence: Vicki Chalker, United Kingdom Health Security Agency, 61 Colindale Ave, London NW9 5EQ, UK; email: Vicki.chalker@phe.gov.uk

\section{Invasive Malaria Vector Anopheles stephensi Mosquitoes in Sudan, 2016-2018}

\author{
Ayman Ahmed, Patricia Pignatelli, Arwa Elaagip, \\ Muzamil M. Abdel Hamid, Omnia Fateh Alrahman, \\ David Weetman \\ Author affiliations: Liverpool School of Tropical Medicine, \\ Liverpool, UK (A. Ahmed, P. Pignatelli, D. Weetman); University \\ of Khartoum, Khartoum, Sudan (A. Ahmed, A. Elaagip, \\ M.M. Abdel Hamid, O. Fateh Alrahman)
}

DOI: https://doi.org/10.3201/eid2711.210400
Anopheles stephensi mosquitoes are urban malaria vectors in Asia that have recently invaded the Horn of Africa. We detected emergence of An. stephensi mosquitoes in 2 noncontiguous states of eastern Sudan. Results of mitochondrial DNA sequencing suggest the possibility of distinct invasions, potentially from a neighboring country.

A nopheles stephensi mosquitoes are efficient vectors of Plasmodium vivax and P. falciparum. Their native range centers on the Indian subcontinent, from which they are increasingly expanding their geographic distribution (1). Recent establishment in Ethiopia (2) and Djibouti (3) is especially worrying. We document the emergence of An. stephensi mosquitoes in Sudan.

Among study sites in a study originally investigating insecticide resistance in the dominant malaria vector in Sudan, Anopheles arabiensis mosquitoes, we selected 12 sites in the eastern half of the country to represent the different ecologic zones (Appendix Figure 1, https://wwwnc.cdc.gov/ EID/article/27/11/21-0400-App1.pdf). We collected Anopheles spp. larvae from all sites in 2016 and from most again in late 2017 or early 2018 (Appendix Table 1). We reared the larvae to adults, checked them morphologically, and initially identified the species as An. gambiae s.l. We extracted DNA from a subset for molecular identification of the species by PCR (3). Of these, 149 DNA samples failed to amplify when we used the standard protocol for identification of the An. gambiae complex, and we investigated them further. We performed mitochondrial cytochrome oxidase 1 amplification and sequencing by using the universal primers C1J-2183 and TL2-N-3014 on the first batch (4); to provide conformity with other studies in East Africa, we used Folmer primers LCO1490 and HCO2198 on a second batch (4). To confirm species identity, we performed BLAST (https://blast.ncbi.nlm. nih.gov/Blast.cgi) searches. We supplemented sequences generated for the mosquitoes from Sudan by using the Folmer primers with sequences from other studies downloaded from GenBank, assembled them by using Clustal within MEGAX (5), and displayed the results as a maximum-likelihood tree with 1,000 bootstraps.

Sequence analysis demonstrated that many of the samples failing diagnostic PCR were not An. gambiae s.l. mosquitoes; most samples identified by BLAST were An. stephensi mosquitoes (Appendix Table 1). The relative frequencies of An. stephensi mosquito detection were similarly high $(>40 \%)$ among those 
from the 2 Red Sea state sites, Port Sudan and Tokar, but seem much lower elsewhere; only 1 individual An. stephensi mosquito was detected at each site in Gedaref state, and none were detected at the other study sites (Appendix Table 1, Figure 2). Although sample site identifications were clear, year identification labels were unfortunately not preserved during sample shipment, and from these samples we cannot determine when An. stephensi mosquitoes were collected. However, sequencing of pools of additional samples from Tokar and Port Sudan from each collection year, which were preserved primarily for RNA analysis, confirmed their presence in both years.
Phylogenetic analysis of the sequences identified 3 haplotypes, which we named Sudan H1-3 (Figure). The most common Sudan haplotype was H3 (86\%), detected at both of the Red Sea state sites (Tokar and Port Sudan); the relatively similar haplotype H2 was detected at lower frequency at Port Sudan only (Appendix Figure 1). H2 and H3 cluster with those collected in Ethiopia in 2016 (2) and with 2 of those collected in 2019, for which we retained the authors' haplotype notation (6). H1 was detected only in Abu Alnaja (Gedaref state) and clustered within a larger clade encompassing a broad range of locations, including a haplotype detected in both Djibouti and Ethiopia (Appendix Figure 1). The sample of

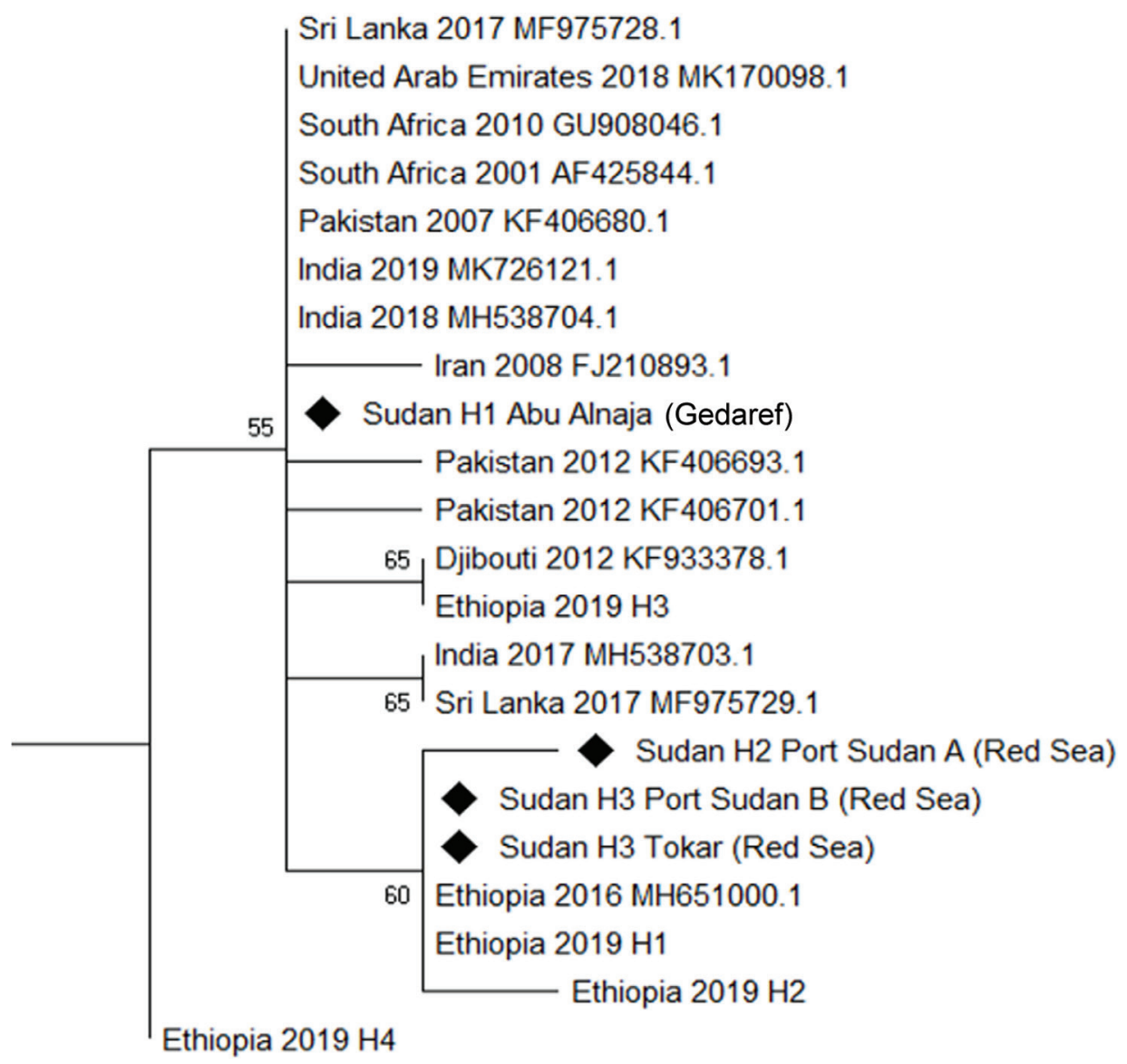

Figure. Phylogenetic analysis of Anopheles stephensi mosquitoes collected in Sudan, 2016-2018, and reference sequences. Maximum-likelihood tree was constructed by using mitochondrial cytochrome oxidase 1 sequences from Sudan (diamonds) and other countries from which data are available. GenBank accession numbers are provided for reference sequences.

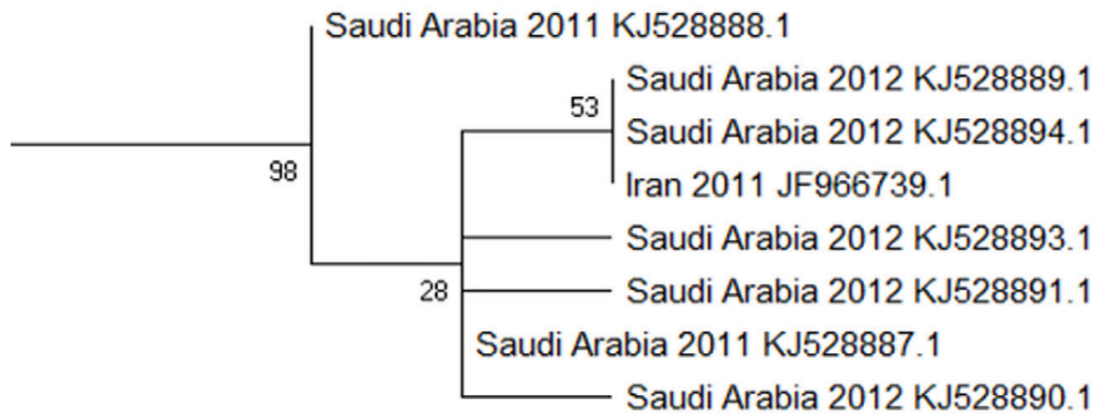


An. stephensi mosquitoes from Daim Bakur failed to amplify after use of the Folmer primers and thus was not included in the tree. The initial origins of $A n$. stephensi mosquitoes are currently difficult to ascertain because of a lack of geographic resolution in the phylogeny; the exception is the highly differentiated clade of samples from Saudi Arabia and Iran. However, the haplotypes detected in Sudan are similar to 3 of the 4 detected in Ethiopia and are thus potentially consistent with spread from Ethiopia or elsewhere in the Horn of Africa. The presence of haplotypes from different states in 2 distinct clades may also indicate separate introduction sources, although wider sampling is required for confirmation.

The emergence of An. stephensi mosquitoes in Sudan poses substantial concern for malaria control and elimination and potentially stark predictions for urban malaria in Africa if this species should spread farther (7). In Sudan and throughout much of Afri$\mathrm{ca}$, local surveillance systems, as well as knowledge and expertise, focus mainly on the mosquito vector members of the predominant An. gambiae complex and An. funestus group (8). Although the actual epidemiologic effects of An. stephensi mosquito emergence is not known, temporal coincidence of their establishment and rising malaria rates in Djibouti suggest a substantial threat (9). Although little is known about $A n$. stephensi mosquitoes in Sudan, we identified productive breeding sites in septic tanks, manholes, and the water-storage containers used for construction purposes in cities (Appendix Figure 3 ); these findings correspond with reports from Ethiopia (6). Information about the wider and local distribution of An. stephensi mosquitoes in Sudan, coupled with bionomic studies, can be used to guide rational control strategies.

\section{Acknowledgments}

We thank our colleagues at the Department of Medical Entomology, Sudan National Public Health Laboratory, who supported and helped us collect and rear the mosquito specimens. We are also grateful to Fitsum Tadesse for providing haplotype sequences from Ethiopia before their publication.

This work was supported by a Wellcome Trust Master's Fellowship in Public Health and Tropical Medicine awarded to A.A. (award no. 200068/Z/15/Z).

\section{About the Author}

Mr. Ahmed is a lecturer at the Institute of Endemic Diseases, University of Khartoum, Sudan, and a Wellcome Trust Master Fellow of Public Health and Tropical Medicine at Liverpool School of Tropical Medicine, UK. His interests are the research and control of the vectorborne diseases, with a particular focus on emerging and re-emerging diseases and invasive disease vectors.

\section{References}

1. Surendran SN, Sivabalakrishnan K, Sivasingham A, Jayadas TTP, Karvannan K, Santhirasegaram S, et al. Anthropogenic factors driving recent range expansion of the malaria vector Anopheles stephensi. Front Public Health. 2019;7:53 https://doi.org/10.3389/fpubh.2019.00053

2. Carter TE, Yared S, Gebresilassie A, Bonnell V, Damodaran L, Lopez K, et al. First detection of Anopheles stephensi Liston, 1901 (Diptera: culicidae) in Ethiopia using molecular and morphological approaches. Acta Trop. 2018;188:180-6. https://doi.org/10.1016/j.actatropica.2018.09.001

3. Scott JA, Brogdon WG, Collins FH. Identification of single specimens of the Anopheles gambiae complex by the polymerase chain reaction. Am J Trop Med Hyg. 1993;49:520-9. https:// doi.org/10.4269/ajtmh.1993.49.520

4. Simon C, Frati F, Beckenbach A, Crespi B, Liu H, Flook P. Evolution, weighting, and phylogenetic utility of mitochondrial gene sequences and a compilation of conserved polymerase chain reaction primers. Ann Entomol Soc Am. 1994;87:651-701. https:/ / doi.org/10.1093/aesa/87.6.651

5. Kumar S, Stecher G, Li M, Knyaz C, Tamura K. MEGA X: Molecular Evolutionary Genetics Analysis across computing platforms. Mol Biol Evol. 2018;35:1547-9. https://doi.org/10.1093/molbev/msy096

6. Tadesse FG, Ashine T, Teka H, Esayas E, Messenger LA, Chali W, et al. Anopheles stephensi mosquitoes as vectors of Plasmodium vivax and falciparum, Horn of Africa, 2019. Emerg Infect Dis. 2021;27:603-7. https:/ / doi.org/10.3201/ eid2702.200019

7. Sinka ME, Pironon S, Massey NC, Longbottom J, Hemingway J, Moyes CL, et al. A new malaria vector in Africa: predicting the expansion range of Anopheles stephensi and identifying the urban populations at risk. Proc Natl Acad Sci U S A. 2020;117:24900-8. https:// doi.org/10.1073/ pnas. 2003976117

8. Takken W, Lindsay S. Increased threat of urban malaria from Anopheles stephensi mosquitoes, Africa. Emerg Infect Dis. 2019;25:1431-3. https:/ /doi.org/10.3201/eid2507.190301

9. de Santi VP, Khaireh BA, Chiniard T, Pradines B, Taudon N, Larréché S, et al. Role of Anopheles stephensi mosquitoes in malaria outbreak, Djibouti, 2019. Emerg Infect Dis. 2021;27:1697-700. https://doi.org/10.3201/eid2706.204557

Address for correspondence: Ayman Ahmed, Institute of Endemic Diseases, University of Khartoum, Khartoum 11111, Sudan; email: ayman.ame.ahmed@gmail.com 